\title{
Imaging and health metrics in Incidental Cerebellar Tonsillar Ectopia: Findings from the Adolescent Brain Cognitive Development Study (ABCD)
}

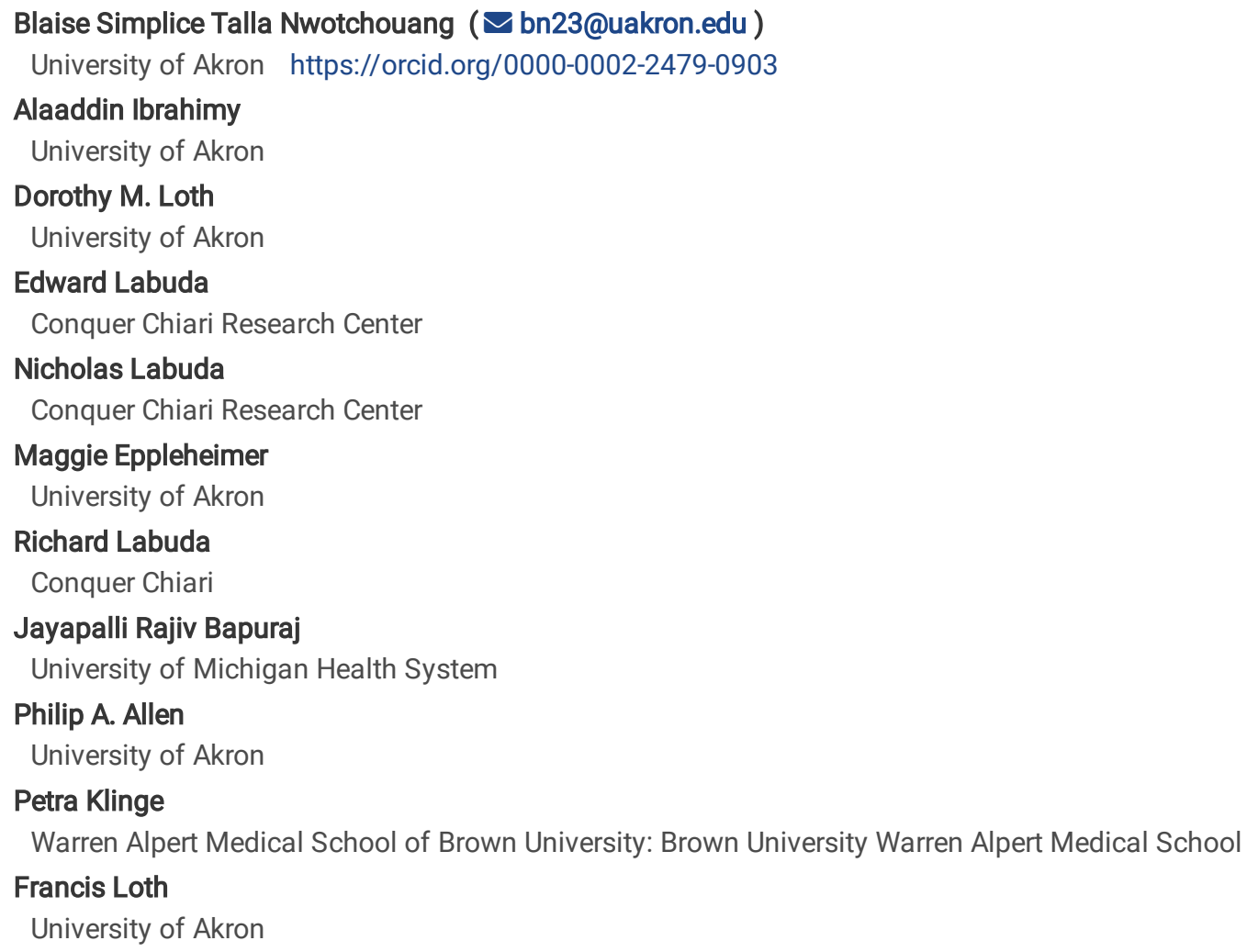

Research Article

Keywords: Chiari malformation type I, incidental cerebellar tonsillar ectopia, Adolescent Brain Cognitive Development (ABCD) pediatric study, magnetic resonance imaging, brain morphometrics

Posted Date: June 30th, 2021

DOI: https://doi.org/10.21203/rs.3.rs-497072/v1

License: @ (i) This work is licensed under a Creative Commons Attribution 4.0 International License. Read Full License

Version of Record: A version of this preprint was published at Neuroradiology on July 11th, 2021. See the published version at https://doi.org/10.1007/s00234-021-02759-y. 


\section{Abstract \\ Purpose.}

Incidental cerebellar tonsillar ectopia (ICTE) that meets the radiographic criterion for Chiari malformation type I (CMI) is an increasingly common finding in the clinical setting, but its significance is unclear. The present study examined posterior cranial fossa (PCF) morphometrics and a broad range of health instruments of pediatric ICTE cases and matched controls extracted from the Adolescent Brain Cognitive Development (ABCD) dataset.

\section{Methods.}

106 subjects with ICTE and 106 matched controls without ICTE were identified from 11,411 anatomical MRI of healthy screened pediatric subjects from the ABCD project. Subjects were matched by sex, age, body mass index, race, and ethnicity. Twenty-two brain morphometrics and 22 health instruments were compared between the two groups to identify unrecognized CMI symptoms and assess the general health impact of ICTE.

\section{Results.}

Twelve and 15 measures were significantly different between the ICTE and control groups for females and males, respectively. Notably, for females, the anterior CSF space was significantly smaller $(p=0.00005)$ for the ICTE group than controls. For males, the clivus bone length was significantly shorter $(p=0.0002)$ for the ICTE group compared to controls. No significant differences were found among the 22 health instruments between the two groups.

\section{Conclusion.}

This study demonstrated that pediatric ICTE subjects have similar PCF morphometrics to adult CMI. ICTE alone did not appear to cause any unrecognized CMI symptoms and had no impact on the subjects' current mental, physical, or behavioral health. Still, given their cranial and brain morphology, these cases may be at risk for adult-onset symptomatic CMI.

\section{Introduction}

The symptomatic impact of pediatric Chiari malformation Type I (CMI) is well documented and includes headaches, trouble sleeping, visual disturbances, auditory issues, and cognitive impairment [1-9]. Incidental cerebellar tonsillar ectopia (ICTE) that meets the radiographic definition of Chiari malformation Type I (CMI) (tonsillar position (TP) $>5 \mathrm{~mm}$ below the McRae line) is an increasingly common finding, especially in children $[10,11]$. Studies of hospital imaging databases have identified the prevalence of ICTE as $1-3.6 \%$, with pediatric rates higher than adults [12-14]. However, ICTE prevalence has been found to be less than $1 \%$ in studies of the general population (0.6-0.9\%) [15, 16]. Pediatric ICTE is generally treated conservatively, with a large majority of pediatric neurosurgeons reporting they choose to monitor for symptomatic and/or neurological changes $[17,18]$. This approach is supported by outcome studies showing that surgical rates over several years from an initial visit for these cases range from 3.5-9.5\% $[10,11,19-21]$ and that new symptoms develop $5-6 \%$ of the time [22].

The impact of ICTE in the pediatric population has yet to be established. The previous pediatric literature of ICTE does not always report truly symptom-free patients. For example, Davidson et al. reported on 218 incidentally discovered CMI cases who were treated conservatively, but more than a third exhibited symptoms at presentation such as seizures $(15.1 \%)$, nonspecific headaches $(14.7 \%)$, or developmental delays (7.8\%). In addition, nearly $10 \%$ of the cases were identified after a trauma.

The Adolescent Brain Cognitive Development Study ${ }^{\circledR}(A B C D)$ is the most extensive long-term study of brain development and child health in the United States, with the goal of creating a unique data resource for the entire scientific community [23]. The dataset contains structural MRI scans acquired in a uniform manner and various mental, physical, and behavioral health metrics and assessments on nearly 12,000 subjects $[24,25]$. Subjects were recruited through schools with the goal of creating a demographically representative sample [26]. Subjects with major medical or neurological conditions, birthweight $<1200$ grams, a history of traumatic brain injury, a current diagnosis of schizophrenia, moderate/severe autism spectrum disorder, intellectual disability, or alcohol/substance use disorder were excluded [27, 28]. As such, the dataset provides an opportunity to study pediatric ICTE cases that were not seeking medical care and are truly incidental.

While the morphometrics of adult $\mathrm{CMI}$ has been studied extensively, less information is available regarding pediatric CMI morphometrics. In fact, several studies have reported the limited availability of MRIs as the reason for excluding pediatric subjects [29-31]. The pediatric 
morphometric studies that exist have produced mixed results, possibly due to the difficulties in acquiring gender and age-matched control images [32-35]. Furtado et al. reported no significant differences in the foramen magnum (FM) area between CMI subjects and controls [32]. However, Trigylidas et al. found significant differences between pediatric $\mathrm{CMI}$ and control subjects for posterior cranial fossa (PCF) volume to intracranial volume ratio and mild differences between asymptomatic and symptomatic CMI subjects [34]. Khalsa et al. examined six midsagittal morphometric and four volumetric measures in 102 pediatric CMI subjects with and without symptoms [33]. They found no significant differences for several brain structures and concluded that no important morphometric measures could accurately distinguish between pediatric subjects with and without CMI-related symptoms [33].

This study utilized the ABCD dataset to answer the following questions:

1. Do adolescents with ICTE have adult-type PCF morphometrics?

2. Does ICTE cause unrecognized CMI symptoms or have a measurable effect on adolescents' physical, mental, and/or behavioral health?

\section{Methods}

\section{Imaging Protocol, Image Selection and Measurements}

T1-weighted images, including the whole brain, were included in this study. Image sets used for this study were acquired on a 3T scanner developed by GE medical system, Siemens Prisma, and Phillips (details of the scanner parameters can be found in Casey et al. [24]). For all the scanners, the images acquired had a pixel size of $1 \times 1 \mathrm{~mm}$, slice thickness $=1 \mathrm{~mm}$, and flip angle $=8$ degrees. In addition, the TR ranged from $6.31 \mathrm{~ms}$ for the Philips scanner to $2500 \mathrm{~ms}$ for Siemens and GE scanners [24]. TE for Siemens, Philips, and GE scanners was $2.88 \mathrm{~ms}, 2.9 \mathrm{~ms}$, and 2 ms, respectively. The FOV for Siemens and GE scanners was 256 x 256, while that of the Philips scanner was 256 x 240 [24]. The number of slices acquired by the Siemens, Philips, and GE scanners was 176, 225, and 208, respectively. A total of 11,411 image sets in Neuroimaging Informatics Technology Initiative (NIfTI) format were acquired from the ABCD Data Repository (NIMH Data Archive). Due to the number of image sets, a protocol was developed to identify ICTE subjects automatically. This process included specialized software to conduct the midsagittal plane's selection and identification of subjects with ICTE. The registration protocol employed the FMRIB Software Library (FSL), developed by the Analysis Group, FMRIB, Oxford, UK, to automatically select the midsagittal images from each patient 3D image set. The MR images were registered using the FSL software library, MATLAB, and each image set was evaluated in a loop using a bash script (Refer to Online Resource 1 for details of the image registration process and the extraction of the midsagittal plane). Using these algorithms, a total of 376 possible ICTE cases were automatically identified.

All automatically identified ICTE cases were manually measured to obtain a TP using MorphPro [35], custom in-house software developed in MATLAB (MathWorks, Natick, MA). The manual measurements revealed 256 of the 376 subjects had TPs $<5$ mm while 120 subjects had TPs greater $>5 \mathrm{~mm}$. A control group was created from the remaining ABCD subjects (TP above McRae line) by matching the ICTE subjects by age, BMI, race, gender, and ethnicity. Race, gender, and ethnicity were matched exactly, including subjects with multiple races. A sufficient match was not found for six of the 120 ICTE subjects, leaving 114 ICTE subjects and 114 matched controls. Next, mid-sagittal images were selected manually on all 228 image sets (114 ICTE and 114 controls) using 3D multi-planar reconstruction in OsiriX (Pixmeo SARL, Switzerland). The software 3D Slicer was used to convert the original downloaded image from NIfTI format to Dicom format. The criterion used to determine the image's midsagittal plane was based on the visibility of at least three of the following four structures: the splenium of the corpus callosum, the pituitary, the cerebral aqueduct, and the genu of the corpus callosum [35]. Eight of 114 incidental cases were found to have a TP less than $5 \mathrm{~mm}$ using the manually obtained midsagittal plane and TP, leaving a total of 106 ICTE subjects and their matched controls for measurement. Nineteen control cases were found to have a TP below the McRae line using the manually obtained midsagittal plane and TP. For these cases, a new control was matched from the ABCD dataset to ensure that all control subjects have TP above the McRae line. All morphometric measurements were conducted by a single operator (DML) with five years of experience in brain morphometric measurements. A second trained operator (AI) with three years of experience in brain morphometric measurements conducted quality control.

Twenty-two 2D measurements were performed by a single operator (DML) using MorphPro [35] and CerePro [36], a custom in-house software developed in MATLAB (MathWorks, Natick, MA) at the University of Akron [35, 36] (Figure 1). The methodology used for the morphometric measures has been described in previous studies [30,31,36]. Each morphometric measurement, briefly described in Table 1, was compared between the ICTE and control groups for both males and females (see Figure 1 for visual examples).

Table 1. Description of morphometric measures. 
Lengths $(\mathrm{mm})$

1) Tonsillar length - the perpendicular distance between the McRae line and the most inferior portion of the tonsils

2) Cerebellar height - Distance between the most superior point of the superior vermis and most inferior point of the tonsil, descended or otherwise

3) Anterior PCF characteristic width - PCF area anterior to brain stem divided by the clivus length

4) Fastigium height - the perpendicular distance between the McRae line and the fastigium of the cerebellum

5) Distance from Pons to McRae - a perpendicular line from the cephalad aspect of the pons at the mid-brain junction to the McRae line

6) Distance from Corpus Callosum to McRae - a perpendicular line from the inferior most aspect of the splenium of the corpus callosum to the McRae line

7) Basioccipital bone length - distance from the synchondrosis to the basion

8) Basisphenoid length - distance from synchondrosis to the top of clivus

9) PCF height - Perpendicular distance from the most anterior portion of the tentorium to the McRaes line

10) Clivus length - distance from the dorsum sellae to the basion

11) McRae Line - the distance calculated from basion to opisthion

12) Intracranial height - the maximum length of the skull perpendicular to the plane of the FM

Areas $\left(\mathrm{mm}^{2}\right)$

13) Anterior PCF area - PCF area anterior to the brain stem

14) PCF area - the area bounded by the tentorium, the occipital bone, the McRae line, and the clivus

15) Cerebellum area in PCF - cerebellum area minus the area of tonsil below the FM represented with " $Y$ " on Figure 1C

16) Cerebellum Area - Area of the cerebellum including tonsils

17) Anterior CSF area - the CSF space in the upper cervical spinal canal anterior to the spinal cord between the FM and the inferior limit of the $\mathrm{C} 2$ vertebrae

18) Posterior CSF area - the CSF space in the upper cervical spinal canal posterior to the spinal cord between the FM and the inferior limit of the $\mathrm{C} 2$ vertebra

Angles $\left({ }^{\circ}\right)$

19) Cerebellar angle with clivus bone length - Angle subtended by the major axis of the cerebellum with the posterior edge of the clivus

20) Boogard Angle - the angle between the clivus and the McRae line

21) Wackenheim angle - formed by a line along the clivus and a line tangent to the posterior aspect of the odontoid process until the base of the $\mathrm{C} 2$ vertebra

22) Odontoid angle - formed by the top of the odontoid process, with the vertex as the midpoint between the anterior-inferior and the posterior-inferior corticated portion of the odontoid, and the posterior-inferior base of the odontoid process

In addition, twenty-two health instruments were selected from the ABCD dataset that represents a broad assessment of physical, mental, and behavioral health (Table 2). From this, a subset of responses (9) that represent established pediatric CMI symptoms - headache, vision problems, auditory issues, seizures, apnea, and cognitive impairment - were selected and compared between the groups to identify unrecognized CMI symptomatology. Finally, the 22 health instruments as a whole were compared between the groups to assess the overall health impact of ICTE.

Table 2. ABCD Health Instruments Selected for Analysis 


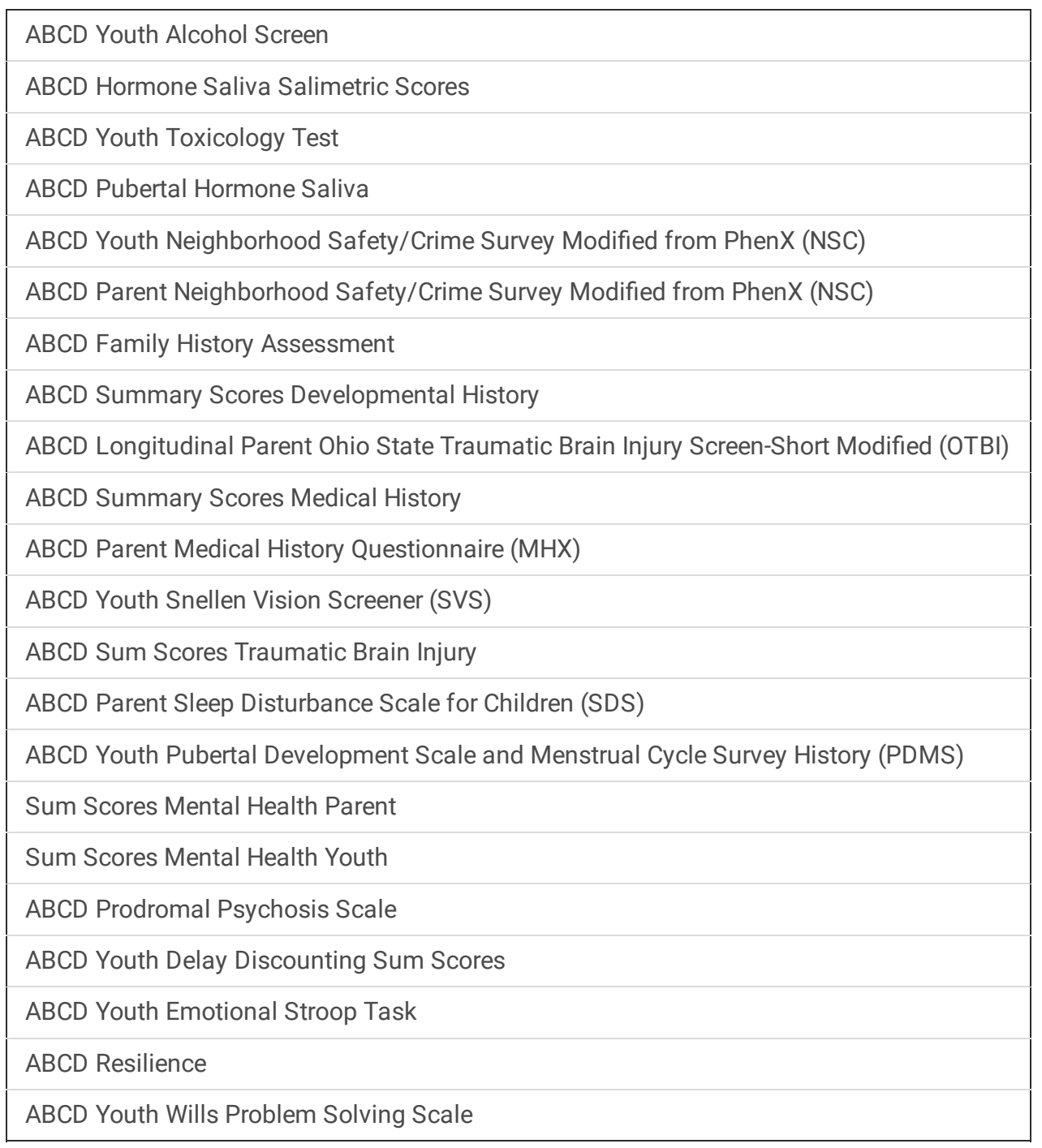

\section{Participants}

This study included 106 ICTE subjects and 106 gender, age, BMI, race, and ethnicity matched pediatric subjects without ICTE (controls). There were 100 females subjects, 50 incidentals (BMI $=19.84 \pm 4.45)$, and 50 controls $(\mathrm{BMI}=19.84 \pm 4.45)$ where both groups had an average age of $9.86 \pm 0.69$ years. There were 112 males subjects, 56 incidentals $(\mathrm{BMI}=18.47 \pm 3.65)$, and 56 controls $(\mathrm{BMI}=18.48 \pm 3.61)$ where both groups had an average age of $9.84 \pm 0.64$ years. Each group comprised $76 \%$ white, $10.4 \%$ Black, and $7.5 \%$ subjects with multiple races. The rest responded as others, did not know, or didn't answer. Ethnically, 16.7\% identified as Hispanic. The anatomical head/neck MRI scans and the physical, mental, and behavioral assessments were obtained from the ABCD data repository [23].

\section{Statistical analysis}

The analysis was divided into three parts: morphometric measures, instruments indicative of unrecognized Chiari symptoms, and all health instruments. Independent student t-tests and Wilcoxon rank-sum tests were used to determine the differences between the incidental and control groups and females and males. For comparison of the instruments between the two groups, categorical data were compared using the Chi-square test, while student's t-test was used to compare quantitative data. The statistical analysis was performed using SPSS and Microsoft Excel. Due to the number of variables compared, we controlled for family-wise errors by applying a Bonferroni correction for multiple comparisons. Statistical significance for adjusted $p$-value was $p<.00022\left(0.05 /\left\{\left[22^{2}-22\right] / 2\right\}\right)$ for the morphometric measures and $p<.0014$ for the CMI symptom subset. Given the extremely large number of comparisons involved in the 22 instruments as a whole, a significance value of $p<.0001$ was selected.

\section{Results}


In females, 12 morphometric measures were significantly different (p's < 0.00022) between the ICTE and control groups, including six linear measures, five areas, and one angle (Table 3). The tonsillar position of the ICTE group was $13 \mathrm{~mm}$ inferior to that of the control group. The distance from the McRae line to the fastigium, pons, and corpus callosum were all about $3 \mathrm{~mm}$ shorter in the ICTE group. The cerebellum height was significantly longer, and the anterior PCF characteristic width was significantly shorter in the ICTE group. The intracranial and PCF heights were each about 3-4 mm shorter in the ICTE group, but this was not statistically significant $(p=0.00048$ and $p=.01372$, respectively). In addition, the McRae line was $1.79 \mathrm{~mm}$ longer for females in the ICTE group, which was not significant $(p=0.00093)$. 
Descriptive statistics of the morphometric measures

\begin{tabular}{|c|c|c|c|c|c|c|c|c|}
\hline $\begin{array}{l}\text { Morphometric } \\
\text { parameter }\end{array}$ & \multicolumn{2}{|c|}{ Incidentals-Mean (SD) } & \multicolumn{2}{|c|}{ Controls-Mean (SD) } & $\begin{array}{l}\text { Female } \\
\text { Incidentals } \\
\text { vs. } \\
\text { Controls }\end{array}$ & $\begin{array}{l}\text { Male } \\
\text { Incidentals } \\
\text { vs. } \\
\text { Controls }\end{array}$ & $\begin{array}{l}\text { Incidentals } \\
\text { Male vs. } \\
\text { Female }\end{array}$ & $\begin{array}{l}\text { Controls } \\
\text { Male vs } \\
\text { Female }\end{array}$ \\
\hline Female & Male & Female & Male & $p$ & $p$ & $p$ & $p$ & $\begin{array}{l}\text { Lengths } \\
(\mathrm{mm})\end{array}$ \\
\hline McRae Line & $36.47(2.66)$ & $38.01(2.63)$ & $34.68(2.59)$ & $37.75(2.72)$ & 0.00093 & 0.60582 & 0.00359 & 0.00000 \\
\hline $\begin{array}{l}\text { Tonsillar } \\
\text { position }\end{array}$ & $9.50(4.75)$ & $8.18(2.57)$ & $-3.48(3.22)$ & $-3.03(3.04)$ & 0.00000 & 0.00000 & 0.18279 & 0.44192 \\
\hline $\begin{array}{l}\text { Fastigium } \\
\text { height }\end{array}$ & $27.04(2.59)$ & $28.80(2.10)$ & $29.85(1.85)$ & $30.82(2.25)$ & 0.00000 & 0.00000 & 0.00026 & 0.01674 \\
\hline $\begin{array}{l}\text { Pons to } \\
\text { McRae }\end{array}$ & $37.34(3.07)$ & $39.27(2.67)$ & $40.23(2.69)$ & $42.19(2.51)$ & 0.00000 & 0.00000 & 0.00090 & 0.00019 \\
\hline $\begin{array}{l}\text { Corpus } \\
\text { Callosum to } \\
\text { McRae }\end{array}$ & $56.94(3.17)$ & $59.14(3.06)$ & $60.16(3.07)$ & $62.14(3.41)$ & 0.00000 & 0.00000 & 0.00044 & 0.00207 \\
\hline $\begin{array}{l}\text { Basioccipital } \\
\text { bone length }\end{array}$ & $25.26(2.54)$ & $24.83(2.14)$ & 26.81(2.79) & $27.26(2.13)$ & 0.02247 & 0.00000 & 0.62825 & 0.42706 \\
\hline $\begin{array}{l}\text { Basisphenoid } \\
\text { length }\end{array}$ & $14.49(1.84)$ & $15.12(2.23)$ & $14.05(1.83)$ & $14.69(1.80)$ & 0.29442 & 0.30661 & 0.15730 & 0.11539 \\
\hline Clivus length & $39.87(2.93)$ & $39.87(3.08)$ & 41.01(3.36) & $42.02(2.82)$ & 0.07247 & 0.00020 & 0.99428 & 0.09899 \\
\hline PCF height & $59.11(4.45)$ & $61.53(4.00)$ & 61.94(4.82) & $64.22(5.43)$ & 0.01372 & 0.00352 & 0.00425 & 0.03480 \\
\hline $\begin{array}{l}\text { Intracranial } \\
\text { height }\end{array}$ & $125.56(5.56)$ & $130.35(4.80)$ & $129.54(4.90)$ & $133.61(4.17)$ & 0.00048 & 0.00021 & 0.00001 & 0.00001 \\
\hline $\begin{array}{l}\text { Cerebellar } \\
\text { height }\end{array}$ & $63.28(5.42)$ & $64.26(3.27)$ & $52.05(4.13)$ & $54.10(4.57)$ & 0.00000 & 0.00000 & 0.05241 & 0.01714 \\
\hline $\begin{array}{l}\text { Anterior PCF } \\
\text { characteristic } \\
\text { width }\end{array}$ & $7.50(1.19)$ & $7.30(1.25)$ & $9.17(1.37)$ & $9.37(1.84)$ & 0.00000 & 0.00000 & 0.41194 & 0.52497 \\
\hline \multicolumn{9}{|l|}{ Areas $\left(\mathrm{mm}^{2}\right)$} \\
\hline PCF area & $3105.48(251)$ & $3262.13(190)$ & $3193.73(283)$ & $3353.44(279)$ & 0.10226 & 0.04533 & 0.00042 & 0.00429 \\
\hline $\begin{array}{l}\text { Anterior PCF } \\
\text { area }\end{array}$ & $299.32(54.95)$ & 291.42(55.19) & $378.20(72.50)$ & $394.80(86.55)$ & 0.00000 & 0.00000 & 0.46296 & 0.28529 \\
\hline $\begin{array}{l}\text { Posterior CSF } \\
\text { area }\end{array}$ & $94.17(38.38)$ & $134.55(44.57)$ & $124.24(37.10)$ & $166.42(43.01)$ & 0.00013 & 0.00008 & 0.00002 & 0.00000 \\
\hline $\begin{array}{l}\text { Anterior CSF } \\
\text { area }\end{array}$ & $105.44(25.00)$ & $115.75(30.09)$ & $129.70(31.57)$ & $123.19(30.51)$ & 0.00005 & 0.33260 & 0.05694 & 0.18072 \\
\hline $\begin{array}{l}\text { Cerebellum } \\
\text { area }\end{array}$ & $1403.18(137)$ & 1518.35(119) & $1178.10(125)$ & $1263.82(160)$ & 0.00000 & 0.00000 & 0.00001 & 0.00257 \\
\hline $\begin{array}{l}\text { Cerebellum } \\
\text { area in PCF }\end{array}$ & $1335.45(128)$ & $1456.25(114)$ & $1178.10(125)$ & $1263.82(160)$ & 0.00000 & 0.00000 & 0.00000 & 0.00257 \\
\hline \multicolumn{9}{|l|}{ Angles $\left({ }^{\circ}\right)$} \\
\hline $\begin{array}{l}\text { Odontoid } \\
\text { angle }\end{array}$ & $71.76(5.02)$ & $72.83(4.79)$ & $73.22(4.88)$ & $73.92(5.01)$ & 0.14509 & 0.24101 & 0.26777 & 0.46687 \\
\hline $\begin{array}{l}\text { Boogard } \\
\text { angle }\end{array}$ & $124.58(7.83)$ & $122.67(6.06)$ & $120.73(5.97)$ & 117.37(4.71) & 0.01107 & 0.00000 & 0.25331 & 0.00472 \\
\hline $\begin{array}{l}\text { Cerebellar } \\
\text { angle with } \\
\text { clivus }\end{array}$ & $25.25(4.60)$ & $22.48(4.21)$ & $20.28(4.57)$ & $16.88(4.95)$ & 0.00000 & 0.00000 & 0.00173 & 0.00037 \\
\hline
\end{tabular}




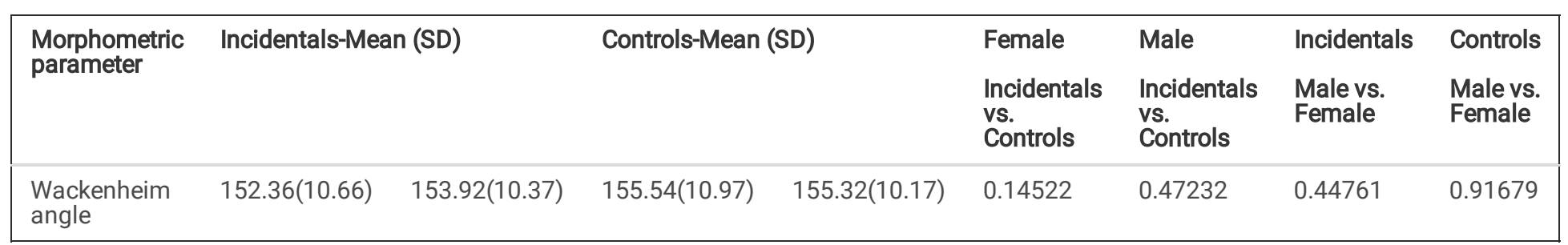

The female ICTE group showed a significant increase in total cerebellum area of about $20 \%$ and a significant increase in PCF cerebellum area of about $15 \%$. This was accompanied by a significant reduction in the anterior PCF area (20\%), anterior CSF area (19\%), and posterior CSF area (24\%). The only angle that was significantly different for the females was the cerebellar angle with clivus, which was about $5^{\circ}$ larger in the ICTE group.

Fifteen measures were significantly different for males in the ICTE group versus controls, including nine linear measures, four areas, and two angles. The tonsillar position was $11 \mathrm{~mm}$ inferior in the ICTE group. The distance from the McRae line to the fastigium, pons, and corpus callosum was 2-3 mm shorter in the ICTE group. The clivus length was $2.15 \mathrm{~mm}$ shorter for the ICTE males. This reduction was comprised entirely of the basioccipital section as no significant difference was found in the length of the basisphenoid. Intracranial height and anterior PCF characteristic width were significantly shorter in the ICTE group, and cerebellar height was significantly longer. PCF height was reduced by about $3 \mathrm{~mm}$ in the ICTE group, but this was not significant $(p=0.00352)$. The cerebellum area and cerebellum area in the PCF were 20 and $15 \%$ larger, respectively, in the ICTE group. The anterior PCF area (26\%) and posterior CSF area (19\%) were smaller in the ICTE group. Additionally, the Boogard angle was $5^{\circ}$ larger on average in the ICTE group than the cerebellar angle with clivus $\left(5.6^{\circ}\right)$.

No significant differences were found for the nine responses representative of CMI symptoms (Table 4 ) or any of the 22 health assessments as a whole between the ICTE and control groups for both sexes or between sexes for either group (all $p>0.001$ ). 
Table 4

Descriptive statistics of the health assessments representative of CMI

\section{Quantitative data}

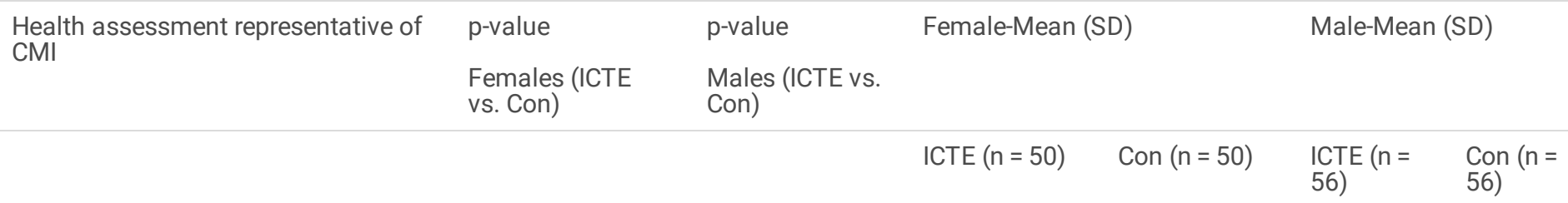

a) Parent Sleep Disturbance Scale

for Children

\begin{tabular}{|c|c|c|c|c|c|c|}
\hline I. Difficulty breathing & 0.32 & 0.77 & $1.02(0.14)$ & $1.0(0.00)$ & $1.05(0.3)$ & $1.08(0.35)$ \\
\hline II. Gasps for breath & 0.32 & 0.32 & $1.0(0.00)$ & $1.03(0.17)$ & $1.02(0.13)$ & $1.0(0.00)$ \\
\hline III. Snores & 0.53 & 1.00 & $1.54(0.89)$ & $1.66(0.8)$ & $1.6(1.01)$ & $1.6(1.01)$ \\
\hline
\end{tabular}

b) Youth Emotional Stroop Task

\begin{tabular}{|c|c|c|c|c|c|c|}
\hline $\begin{array}{l}\text { I. Proportion correct overall (across } \\
\text { all test trials) }\end{array}$ & 0.41 & 0.87 & $0.96(0.03)$ & $0.97(0.03)$ & $0.94(0.05)$ & $0.94(0.08)$ \\
\hline c) Youth Wills Problem Solving Scale & 0.78 & 0.33 & $23.6(4.5)$ & $23.2(4.5)$ & $23.6(5.1)$ & $25.0(3.7)$ \\
\hline
\end{tabular}

Categorical data ( 1 or 0 )

$\begin{array}{ll}\text { p-value } & \text { p-value } \\ \text { Females (ICTE vs } & \begin{array}{l}\text { males (ICTE vs } \\ \text { Con) }\end{array}\end{array}$

№ of female subjects without № of male subjects symptom

without symptom

$\operatorname{ICTE}(n=50) \quad$ Con $(n=50) \quad \operatorname{ICTE}(n=\quad$ Con $(n=$

56) 56)

d) Parent History Medical

Questionnaire

$\begin{array}{llllll}\text { I. Hearing }(1,0) & 0.05 & 0.05 & 49 & 46 & 56 \\ \text { II. Vision }(1,0) & 0.7 & 0.8 & 35 & 38 & 51 \\ \text { III. Bad Headaches }(1,0) & 0.8 & 0.2 & 48 & 49 & 51\end{array}$

a) Summary Scores Medical History

I. Seizures $(1,0)$

0.3

0.16

49

50

54

56

\section{Discussion}

For the first time, this study examined PCF morphometrics and the overall health impact of ICTE in the general pediatric population. Previous pediatric morphometrics studies have been limited in terms of both the morphometrics measures examined and how subjects were selected [32-34]. Perhaps because of this, results have been mixed. In comparing CMI to controls, Trigylidas et al. [34] found differences, while Furtado et al. did not [32]. Khalsa et al. compared asymptomatic to symptomatic CMI across ten morphometrics measures and found no differences, implying that structurally ICTE may be similar to symptomatic CMI. However, Khalsa et al. used hospital-based images, which raises the question of whether the finding of TP $>5 \mathrm{~mm}$ is strictly incidental since each patient presented at the hospital with sufficient concern to justify head and neck MRI acquisition [33]. The present study used the ABCD data repository to identify the ICTE and control pediatric subjects. All ABCD participants were screened for major health and neurological issues, ensuring that all subjects were healthy, and thus, all subjects found with TP > 5mm were strictly incidental. In addition, the size of the ABCD repository enabled the selection of a carefully matched control group by race, ethnicity, gender, age, BMI, all factors that can affect morphometric evaluation [37]. Finally, 22 morphometrics measures were selected representing cranial, tissue, PCF lengths, areas, and angles that together comprise an extensive assessment of the PCF morphometrics. This incidental tonsillar ectopia study is highly unique because of the careful screening of the $A B C D$ participants, the fact that the $A B C D$ participants were volunteering and not seeking medical care, and because the control group was precisely matched to the ICTE group.

\section{PCF Morphometrics}


The present pediatric results were compared with adult CMI PCF morphometrics reported previously [31, 36, 37]. Specifically, Houston et al. looked at > 115 adult female CMI subjects compared to matched controls and found 14 significant differences they grouped as PCF structure heights, clivus angulation, and odontoid process irregularities [31, 36]. In a smaller follow-up study, Houston et al. examined the same PCF morphometrics in 26 male symptomatic CMI subjects and matched controls and found largely similar results as with females, but that the male differences were larger in magnitude [37]. In a study using many of the same subjects as Houston et al., Biswas et al. found that adult female CMI subjects exhibited larger cerebellum (in the PCF space) and reduced CSF spaces below the FM [36]. Our study has found pediatric ICTE subjects, both male and female, demonstrate similar differences to controls as adult symptomatic CMI cases do in terms of reduced PCF structure heights and crowding, but not in terms of clivus angulation or odontoid process irregularities. In addition, we found several genderbased differences in comparing ICTEs to controls.

\section{PCF Structure Heights}

Houston et al. found that in adults (females and males), the distances from the fastigium, pons, and corpus callosum to the McRae line were $\sim 3 \mathrm{~mm}$ shorter in CMI subjects compared to controls, meaning the entire hindbrain sits lower [31, 37]. Pediatric ICTE subjects were also found to have a $\sim 3 \mathrm{~mm}$ shortening of the distance from the McRae line to these hindbrain structures, implying a similar type of hindbrain sagging as seen in adult CMI. However, the clivus bone in adult CMI has also been found to be significantly shorter compared to controls, with a larger reduction in men than women (5.4 vs. $3 \mathrm{~mm}$, respectively) [29, 31, 37]. In the present study, the differences in clivus length between ICTE and controls were not as dramatic (1.15 and $2.15 \mathrm{~mm}$, for females and males, respectively), with only the male difference reaching statistical significance. This could be an indication of ICTE versus symptomatic CMI in females as it is similar to what Nwotchouang et al. found in comparing symptom-free female adults with low-lying tonsils (TP $<5 \mathrm{~mm}$ ) to healthy controls. They reported that the clivus lengths were similar between the two groups $(0.3 \mathrm{~mm}$ difference, $p=0.79$ [38]. However, it could also be due to the relatively young age of the ICTE subjects, as the closure of the synchondrosis between the basisphenoid and basioccipital regions of the clivus does not start in girls until $12-13$ years, in boys at 14-15 years, and is not complete until 17-18 years [39, 40].

\section{Crowding}

Compared to controls, adult female CMI patients have been found to have a significantly smaller PCF area on average combined with an 8.4\% larger cerebellum (even without considering the herniated tonsils), resulting in crowding of the PCF [36]. In addition, compared to controls, adult female CMI have reduced CSF spaces below the FM (25 and 20\% on the posterior and anterior sides, respectively). Unfortunately, similar morphometrics measurements are not available for adult male CMI subjects in the literature. We found that both female and male pediatric ICTE subjects showed significant reductions in anterior PCF and PCF areas relative to controls and increased cerebellum area inside the PCF of 13 and $15 \%$, respectively. While this increase in cerebellum size is larger than seen in adults, it should be noted that total cerebellar volume has been found to follow an inverted $U$ course with age, peaking at around 12 years for females and 15-16 years for males [41]. Regardless, both male and female ICTE subjects clearly showed crowding of the PCF area similar to what is seen in symptomatic adult CMI.

In terms of crowding below the FM, the posterior CSF space was significantly reduced by $24 \%$ for pediatric ICTE females and $19 \%$ for pediatric ICTE males compared to controls, similar to the adult female CMI finding. However, the anterior CSF space was only significantly reduced for ICTE females (19\%) and not males. Since this morphometric is not available for adult male CMI, this finding's implications are not clear. It is interesting to note that it could contribute to the fact that females far outnumber males in adult symptomatic CMI prevalence [42-44].

\section{Clivus Angulation}

Adult CMI subjects on average demonstrate a flattening of the clivus bone indicative of cranial settling as reflected in a significant increase in the Boogard angle ( $3^{\circ}$ for females, $6^{\circ}$ for males) and a significant decrease in the Wackenheim angle $\left(6^{\circ}\right.$ for females, $8^{\circ}$ for males) [31, 37]. For this study, the Boogard angle showed similar differences between the ICTE and control groups ( $4^{\circ}$ for females and $5^{\circ}$ for males), but the Wackenheim angle did not ( $3^{\circ}$ for females and $1^{\circ}$ for males). This may be due to the age of the subjects, but it also could have implications for the pathogenesis of CMI symptoms. Since Boogard angle and Wackenheim angle overlap via the clivus, this implies that the alignment of the odontoid, C1 and C2 may be different in adult symptomatic CMI compared to incidental cases. This is supported by the fact that Houston et al. also found that the odontoid angle was significantly smaller in adult female $\mathrm{CMI}$ compared to controls $\left(3^{\circ}\right)$, but in this study, the odontoid angle was not significantly different between the ICTE groups and controls. It could be that misalignment of the upper cervical spine of even just a few degrees plays a role in adult CMI symptomatology, perhaps through subtle instability. Goel et al. has recently proposed that cervical instability plays a primary role in CMI symptoms and that the cerebellar tonsils herniate to cushion from mechanical pinching, but his theory does not account for the preponderance of ICTE compared to symptomatic CMI [45]. It seems more likely that upper cervical misalignment, even if subtle, is one of several factors which, in concert, contribute to the development of CMI symptoms.

\section{Normative Values}


Although the control subject group was not selected randomly or with purposeful distribution by race and ethnicity, it still represents a large sample of healthy 9-10 year-olds. As such, this study established for the first-time normative PCF morphometric values for that age range in both males and females, which can be used as the basis for future comparative studies.

\section{Health Metrics}

No significant differences were found between either female or male ICTE and the associated control groups for any of the physical, mental and behavioral health instruments. Given the breadth of assessments, it is reasonable to conclude that ICTE in and of itself does not have a measurable impact on the overall health of 9-10 year old pediatric subjects.

In addition, the health instruments contained a number of elements that would be indicative of unrecognized CMI symptoms. Headaches have been found to affect $40-78 \%$ of pediatric symptomatic CMI cases, yet the Medical History Questionnaire revealed no significant difference between either the male or female ICTE groups and the associated control groups in terms of headache history (Table

4) $[1,5]$. Similarly, both vision problems - such as nystagmus, strabismus, diplopia, and blurred vision - and sensorineural hearing loss have been linked to pediatric CMI, but again, the Medical History Questionnaire showed no difference between the ICTE and control groups [3, 6, 9] in these areas. Although less common, seizures have also been linked by some to pediatric CMI, but the Medical History showed no differences in this regard either $[46,47]$.

The health instruments included a Parent Sleep Disturbance Scale for Children. Using polysomnography, Amin et al. found that $49 \%$ of 68 pediatric $\mathrm{CMI}$ cases exhibited sleep-disordered breathing. In a review, Abel et al. and Tahir et al. concluded that sleep-disordered breathing is highly prevalent in pediatric CMI $[2,8]$. In contrast, we found no significant difference in reports of difficulty breathing during sleep, gasping for air, snoring, or overall sleep patterns between the ICTE and control groups.

Both parent surveys and cognitive testing have identified that CMI can impair executive function in pediatric cases [7, 48]. We found no significant differences in either the Stroop Test or Youth Wills Problem Solving Scale between the pediatric ICTE and control groups.

Finally, although the prevalence has not been established, pediatric CMI has been linked to precocious puberty in both males and females $[48,49]$. Given the focus of the ABCD study, it includes both a Pubertal Development/Menstrual Cycle Survey and a hormonal assay. Again, no significant differences were found between the ICTE and control groups for these measures.

It is important to consider that it is unknown if any of the ICTE cases will manifest symptoms later in childhood or as adults. In a natural history review, Chatrath et al. found that 5-6\% of clinically identified pediatric asymptomatic CMI cases develop symptoms over the course of several years [22]. However, no study has followed pediatric ICTE cases into adulthood, and given their morphology, the adolescents identified here may be at risk for adult-onset CMI.

\section{ICTE Prevalence}

Because the false-negative rate for the methodology used to identify the ICTE subjects from the ABCD repository is unknown, establishing an accurate ICTE prevalence from this study is not possible. However, since the automatic results were manually screened for false positives, a lower bound for ICTE prevalence among 9 to 10 year olds can be determined confidently. We identified and confirmed 112 ICTE subjects out of 11,411, representing a lower bound of $0.98 \%$. This is lower than Smith et al. found, but they utilized a hospital database as opposed to a general pediatric population [14]. A manual review of the entire ABCD repository would be required to specify the exact ICTE prevalence, but such an undertaking was beyond the scope of this study.

\section{ICTE \& Symptomatic CMI}

Radiographically, the ICTE groups in this study looked remarkably similar to adult symptomatic CMI cases. The average TP for females was $9.5 \mathrm{~mm}$ and for males was $8.2 \mathrm{~mm}$. In addition, there were 20 cases with TP > $10 \mathrm{~mm}$ and 4 cases with TP > $15 \mathrm{~mm}$. ICTE subjects showed a reduced height of PCF structures, crowding inside the PCF, and reduced CSF spaces below the FM. Despite this, there were no indications of any unrecognized CMI symptoms, or health impact in general, of this anatomy. This calls into question the utility of TP and crowding as indicators of CMI and also the theory that symptoms arise from a mass effect and CSF restriction [50]. However, as opposed to adult CMI, the ICTE cases did show normal alignment of the upper cervical spine and odontoid. This could mean that misalignment of C1, C2, and the odontoid process plays a role in CMI symptomatology, but further research is required to establish this.

It is also possible that a subset of the identified pediatric ICTE cases will go on to develop CMI symptoms as adults. However, the prevalence of symptomatic CMI has been estimated at $0.1 \%$ [51]. The prevalence of ICTE, even among adults, is an order of magnitude higher; so how likely it is for an individual pediatric ICTE subject to develop adult-onset Chiari is not clear. In this scenario, it is also not clear why symptoms manifest in adulthood. It could be due to aging issues further affecting the PCF morphology, or it is possible that morphometrics 
such as TP and PCF/CSF crowding are a necessary but not sufficient component of symptomatic CMI and that physical trauma or dynamic factors, such as involving the vascular and/or CSF systems, play a role. Either way, adult CMI PCF morphometrics in adolescence is a potential risk factor for adult-onset CMI.

The ABCD study intends to follow subjects ten years into early adulthood with repeated imaging and health assessments. This provides an unprecedented opportunity to track the PCF morphological development of the ICTE and control groups as they mature. The assessments can also be used to monitor the development of any impact ICTE may have on the subjects' mental, physical, or behavioral health.

This study's primary limitation lies in interpreting the results of the morphometric differences found between pediatric ICTE and controls compared to established morphometric differences between adult CMI and controls. Ideally, a third matched group of pediatric CMI would be included for analysis rather than relying on adults. But identifying precisely matched CMI subjects in sufficient numbers was impossible. This means that the variations in the pediatric differences compared to the adult differences could be due to age and not necessarily reflective of symptomatic CMI. However, over time as repeated studies are performed on the same ICTE and control subjects into adulthood, this limitation will be mitigated. A second limitation arises from using an automated process to identify the ICTE subjects from the ABCD repository. Although the identified ICTE cases were manually verified, it is not known what portion of the total ICTE cases in the repository they represent. Some ICTE cases were likely missed, and as a result, sampling bias could have been introduced as a result of the selection program used.

\section{Conclusions}

This study extracted truly asymptomatic pediatric ICTE subjects from the ABCD database and examined their PCF morphometrics and overall health compared to carefully matched controls. The identified ICTE group showed similar morphometrics to adult CMI in terms of the height of PCF structures and crowding of the PCF and CSF spaces below the FM, but not in terms of clivus and odontoid angulation. Despite the morphometric similarities to CMI, ICTE had no apparent impact on the current health of the subjects as assessed across mental, physical, and behavioral measures. The findings imply that adult-type morphometrics in pediatric ICTE might be a biomarker to identify a risk to develop adult-onset symptomatic CMI.

\section{Declarations}

\section{Compliance with ethical standards}

Funding: The study was funded by Conquer Chiari

Ethical Approval:

This study was approved by the local institutional review board at The University of Akron. All procedures performed in studies involving human participants were in accordance with the ethical standards of the institutional and/or national research committee and with the 1964 Helsinki declaration and its later amendments or comparable ethical standards. For this type of study formal consent is not required.

Informed consent: Informed consent was obtained from all individual participants included in the study.

Conflict of Interest Disclosure: Author Jayapalli Rajiv Bapuraj is a recipient of a research grant from Conquer Chiari.

Acknowledgements: Data used in the preparation of this article were obtained from the Adolescent Brain Cognitive Development (ABCD) Study (https://abcdstudy.org), held in the NIMH Data Archive (NDA). This is a multisite, longitudinal study designed to recruit more than 10,000 children age 9-10 and follow them over 10 years into early adulthood. The ABCD Study is supported by the National Institutes of Health and additional federal partners under award numbers U01DA041048, U01DA050989, U01DA051016, U01DA041022, U01DA051018, U01DA051037, U01DA050987, U01DA041174, U01DA041106, U01DA041117, U01DA041028, U01DA041134, U01DA050988, U01DA051039, U01DA041156, U01DA041025, U01DA041120, U01DA051038, U01DA041148, U01DA041093, U01DA041089, U24DA041123, U24DA041147. A full list of supporters is available at https://abcdstudy.org/federal-partners.html. A listing of participating sites and a complete listing of the study investigators can be found at https://abcdstudy.org/consortium_members/. ABCD consortium investigators designed and implemented the study and/or provided data but did not necessarily participate in analysis or writing of this report. This manuscript reflects the views of the authors and may not reflect the opinions or views of the NIH or ABCD consortium investigators

The ABCD data repository grows and changes over time. The ABCD data used in this report came from the fast track data release. The raw data are available at https://nda.nih.gov/edit_collection.html?id=2573.

\section{References}


1. Chiari C (2021) [cited 2021 23-Apr-2021]; Available from: https://chiari1000.uakron.edu/

2. Amin R et al (2015) The association between sleep-disordered breathing and magnetic resonance imaging findings in a pediatric cohort with Chiari 1 malformation. Can Respir J 22(1):31-36

3. Kumar A, Patni AH, Charbel F (2002) The Chiari I malformation and the neurotologist. Otol Neurotol 23(5):727-735

4. Sari SA, Ozum U (2021) The executive functions, intellectual capacity, and psychiatric disorders in adolescents with Chiari malformation type 1. Childs Nerv Syst

5. Tubbs RS et al (2011) Institutional experience with 500 cases of surgically treated pediatric Chiari malformation Type I. J Neurosurg Pediatr 7(3):248-256

6. Shaikh AG, Ghasia FF (2015) Neuro-ophthalmology of type 1 Chiari malformation. Expert Rev Ophthalmol 10(4):351-357

7. Lacy M et al (2016) Parent-Reported Executive Dysfunction in Children and Adolescents with Chiari Malformation Type 1. Pediatr Neurosurg 51(5):236-243

8. Abel F, Tahir MZ (2019) Role of sleep study in children with Chiari malformation and sleep disordered breathing. Childs Nerv Syst 35(10):1763-1768

9. Simons JP, Ruscetta MN, Chi DH (2008) Sensorineural hearing impairment in children with Chiari I malformation. Ann Otol Rhinol Laryngol 117(6):443-447

10. Davidson L et al (2021) Long-term outcomes for children with an incidentally discovered Chiari malformation type 1: what is the clinical significance? Childs Nerv Syst 37(4):1191-1197

11. Whitson WJ et al (2015) A prospective natural history study of nonoperatively managed Chiari I malformation: does follow-up MRI surveillance alter surgical decision making? J Neurosurg Pediatr 16(2):159-166

12. Meadows J et al (2000) Asymptomatic Chiari Type I malformations identified on magnetic resonance imaging. J Neurosurg 92(6):920926

13. O'Reilly EM, Torreggiani W (2019) Incidence of Asymptomatic Chiari Malformation. Ir Med J 112(7):972

14. Smith BW et al (2013) Distribution of cerebellar tonsil position: implications for understanding Chiari malformation. J Neurosurg $119(3): 812-819$

15. Jansen PR et al (2017) Incidental Findings on Brain Imaging in the General Pediatric Population. N Engl J Med 377(16):1593-1595

16. Vernooij MW et al (2007) Incidental findings on brain MRI in the general population. N Engl J Med 357(18):1821-1828

17. Haroun RI et al (2000) Current opinions for the treatment of syringomyelia and chiari malformations: survey of the Pediatric Section of the American Association of Neurological Surgeons. Pediatr Neurosurg 33(6):311-317

18. Singhal A, Cheong A, Steinbok P (2018) International survey on the management of Chiari 1 malformation and syringomyelia: evolving worldwide opinions. Childs Nerv Syst 34(6):1177-1182

19. Benglis D Jr et al (2011) Outcomes in pediatric patients with Chiari malformation Type I followed up without surgery. J Neurosurg Pediatr 7(4):375-379

20. Leon TJ et al (2019) Patients with "benign" Chiari I malformations require surgical decompression at a low rate. J Neurosurg Pediatr 23(4):498-506

21. Strahle $\mathrm{J}$ et al (2011) Natural history of Chiari malformation Type I following decision for conservative treatment. J Neurosurg Pediatr $8(2): 214-221$

22. Chatrath A et al (2019) Chiari I malformation in children-the natural history. Childs Nerv Syst 35(10):1793-1799

23. Adolescent Brain Cognitive Development (ABCD) 2021 [cited 2021 02/24/2021]; Available from: https://abcdstudy.org/about/

24. Casey BJ et al (2018) The Adolescent Brain Cognitive Development (ABCD) study: Imaging acquisition across 21 sites. Dev Cogn Neurosci $32: 43-54$

25. Barch DM et al (2018) Demographic, physical and mental health assessments in the adolescent brain and cognitive development study: Rationale and description. Dev Cogn Neurosci 32:55-66

26. Garavan $\mathrm{H}$ et al (2018) Recruiting the ABCD sample: Design considerations and procedures. Dev Cogn Neurosci 32:16-22

27. Michelini G et al (2019) Delineating and validating higher-order dimensions of psychopathology in the Adolescent Brain Cognitive Development (ABCD) study. Transl Psychiatry 9(1):261

28. Li Y et al (2021) Rates of Incidental Findings in Brain Magnetic Resonance Imaging in Children. JAMA Neurol

29. Nwotchouang BST et al (2019) Three-Dimensional CT Morphometric Image Analysis of the Clivus and Sphenoid Sinus in Chiari Malformation Type I. Annals of Biomedical Engineering

30. Eppelheimer MS et al (2019) Quantification of changes in brain morphology following posterior fossa decompression surgery in women treated for Chiari malformation type 1. Neuroradiology 61(9):1011-1022

Page 13/15 
31. Houston JR et al (2018) A morphometric assessment of type I Chiari malformation above the McRae line: A retrospective case-control study in 302 adult female subjects. J Neuroradiol 45(1):23-31

32. Furtado SV et al., Morphometric analysis of foramen magnum dimensions and intracranial volume in pediatric Chiari I malformation. Acta Neurochir (Wien), 2010. 152(2): p. 221-7; discussion 227

33. Khalsa SSS et al (2018) Morphometric and volumetric comparison of 102 children with symptomatic and asymptomatic Chiari malformation Type I. J Neurosurg Pediatr 21(1):65-71

34. Trigylidas T et al (2008) Posterior fossa dimension and volume estimates in pediatric patients with Chiari I malformations. Childs Nerv Syst 24(3):329-336

35. Eppelheimer MS et al (2018) A Retrospective 2D Morphometric Analysis of Adult Female Chiari Type I Patients with Commonly Reported and Related Conditions. Front Neuroanat 12:2

36. Biswas D et al (2019) Quantification of Cerebellar Crowding in Type I Chiari Malformation. Ann Biomed Eng 47(3):731-743

37. Houston JR et al (2020) Evidence of Neural Microstructure Abnormalities in Type I Chiari Malformation: Associations Among Fiber Tract Integrity, Pain, and Cognitive Dysfunction. Pain Med 21(10):2323-2335

38. Nwotchouang BST et al (2020) Clivus length distinguishes between asymptomatic healthy controls and symptomatic adult women with Chiari malformation type I. Neuroradiology 62(11):1389-1400

39. Krishan K, Kanchan T (2013) Evaluation of spheno-occipital synchondrosis: A review of literature and considerations from forensic anthropologic point of view. J Forensic Dent Sci 5(2):72-76

40. Bassed RB, Briggs C, Drummer OH (2010) Analysis of time of closure of the spheno-occipital synchondrosis using computed tomography. Forensic Sci Int 200(1-3):161-164

41. Tiemeier $\mathrm{H}$ et al (2010) Cerebellum development during childhood and adolescence: a longitudinal morphometric MRI study. Neuroimage 49(1):63-70

42. Wilkinson DA et al (2017) Trends in surgical treatment of Chiari malformation Type I in the United States. J Neurosurg Pediatr 19(2):208216

43. Greenberg JK et al (2015) Complications and Resource Use Associated With Surgery for Chiari Malformation Type 1 in Adults: A Population Perspective. Neurosurgery 77(2):261-268

44. Marty P et al., Gender-specific differences in adult type I Chiari malformation morphometrics (P4.174). 2015. 84(14 Supplement): p. P4.174

45. Goel A et al (2020) Chiari 1 Formation Redefined-Clinical and Radiographic Observations in 388 Surgically Treated Patients. World Neurosurg 141:e921-e934

46. Brill CB, Gutierrez J, Mishkin MM (1997) Chiari I malformation: association with seizures and developmental disabilities. J Child Neurol 12(2):101-106

47. Granata T, Valentini LG (2011) Epilepsy in type 1 Chiari malformation. Neurol Sci 32(Suppl 3):S303-S306

48. Stagi S et al (2004) Precocious, early and fast puberty in males with Chiari I malformation. J Pediatr Endocrinol Metab 17(8):1137-1140

49. Pucarelli I et al (2010) Precocious puberty in two girls with Chiari I malformation: a contribution to a larger use of brain MRI in the diagnosis of central precocious puberty. Minerva Pediatr 62(3):315-317

50. Bolognese PA et al (2019) Chiari I Malformation: Opinions on Diagnostic Trends and Controversies from a Panel of 63 International Experts. World Neurosurgery 130:e9-e16

51. Sadler B et al (2020) Prevalence and Impact of Underlying Diagnosis and Comorbidities on Chiari 1 Malformation. Pediatr Neurol 106:3237

\section{Figures}



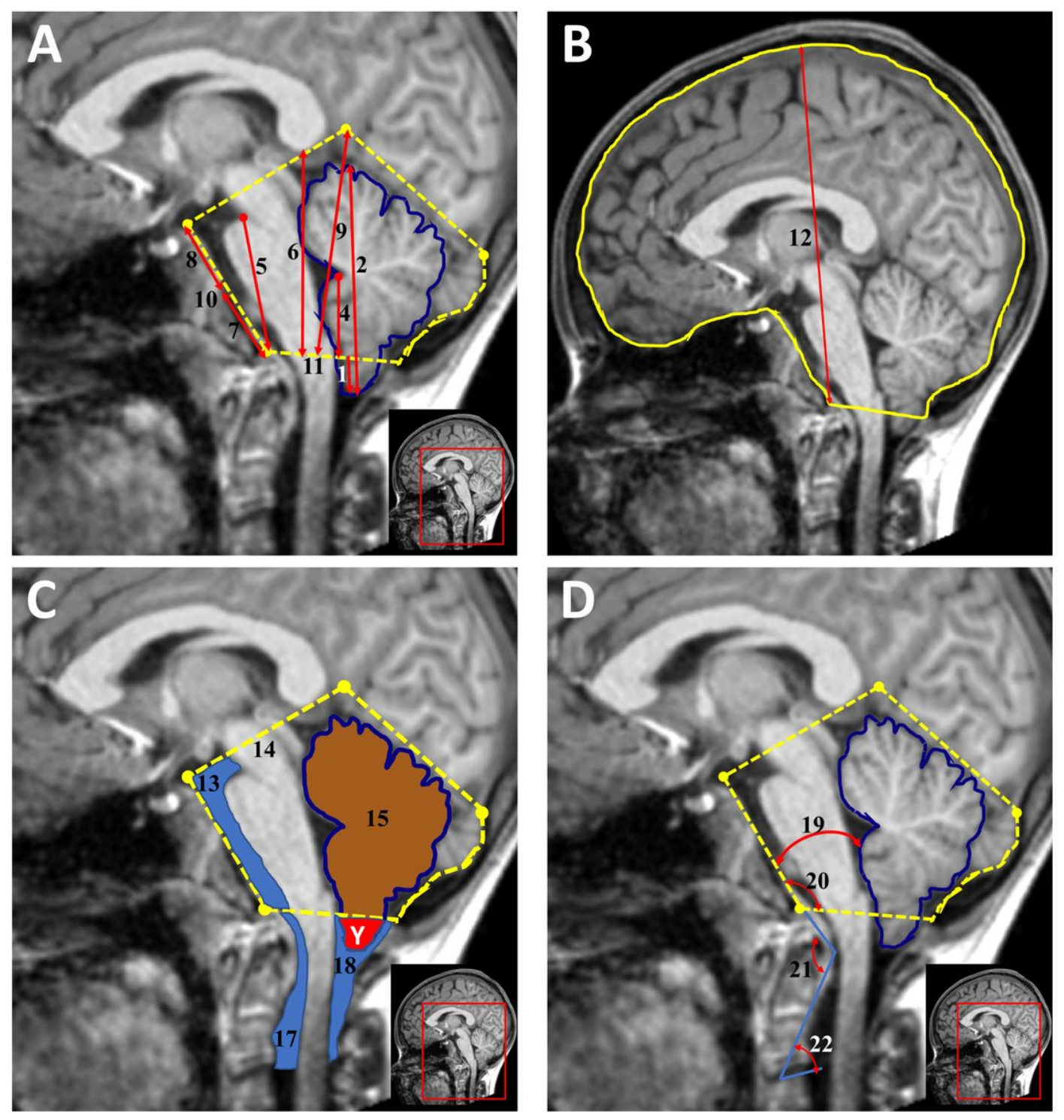

Figure 1

Morphometric Measures: (1) Tonsillar length, (2) Cerebellar height, (3) Anterior PCF characteristic width (anterior PCF area \#13/ clivus length \#10, (4) Fastigium height, (5) Distance from Pons to McRae, (6) Distance from Corpus Callosum to McRae, (7) Basioccipital bone length, (8) Basisphenoid length, (9) PCF height, (10) Clivus length, (11) McRae Line, (12) Intracranial height, (13) Anterior PCF area, (14) PCF area, (15) Cerebellum area in PCF, (16) Cerebellum Area (\#15 + the tonsillar area identified in white and marked as "Y" on Figure 1C), (17) Anterior CSF area, (18) Posterior CSF area, (19) Cerebellar angle with clivus bone length, (20) Boogard Angle, (21) Wackenheim angle, (22) Odontoid angle.

\section{Supplementary Files}

This is a list of supplementary files associated with this preprint. Click to download.

- ESM1.pdf 